\title{
Optimization-Based Evolutionary Data Mining Techniques for Structural Health Monitoring
}

\author{
Meisam Gordan, Zubaidah Binti Ismail, Hashim Abdul Razak, Khaled Ghaedi, \\ Haider Hamad Ghayeb \\ Department of Civil Engineering, University of Malaya, 50603, Kuala Lumpur, Malaysia \\ E-mail: meisam.gordan@gmail.com
}

Received: 27 July 2019; Accepted: 24 August 2019; Available online: 20 November 2019

\begin{abstract}
In recent years, data mining technology has been employed to solve various Structural Health Monitoring (SHM) problems as a comprehensive strategy because of its computational capability. Optimization is one the most important functions in Data mining. In an engineering optimization problem, it is not easy to find an exact solution. In this regard, evolutionary techniques have been applied as a part of procedure of achieving the exact solution. Therefore, various metaheuristic algorithms have been developed to solve a variety of engineering optimization problems in SHM. This study presents the most applicable as well as effective evolutionary techniques used in structural damage identification. To this end, a brief overview of metaheuristic techniques is discussed in this paper. Then the most applicable optimization-based algorithms in structural damage identification are presented, i.e. Particle Swarm Optimization (PSO), Genetic Algorithm (GA), Imperialist Competitive Algorithm (ICA) and Ant Colony Optimization (ACO). Some related examples are also detailed in order to indicate the efficiency of these algorithms.
\end{abstract}

Keywords: Structural damage detection; Data mining; Metaheuristic algorithms; Optimization.

\section{Introduction}

In-service aerospace, mechanical, and civil structures are damage-prone during their service life. This is due to the fact that different external loads induced by wind excitations, earthquakes and other natural disasters can disturb the serviceability of these structures. Therefore, the external loads can cause structural damage and out-ofservice conditions which can change the structural properties, i.e. stiffness, damping, and mass and finally change in the dynamic characteristics of structures (e.g. natural frequency, mode shape and damping ratio). To overcome such problems, SHM is applied as an important strategy in order to guarantee the integrity, safety, and serviceability of structures [1-4].

A SHM methodology can be implemented in two parts, i.e. data collection and data extraction. The response data is collected in the first stage using a number of sensors attached to the structure. Then after data creation by monitoring system, substantial knowledge concerning the structural health condition are obtained in the second part from raw databases [5,6]. Nowadays, several computing and information technologies exist, e.g. data mining, evolutionary computing and knowledge engineering which have gained significant attention is civil engineering. Consequently, civil engineers have embraced computer science technology in the last decades [7]. Data mining is applied to evaluate the unprocessed datasets in order to discover the best relationship among inputs and outputs [8]. Hence, data mining can provide a possible solution for structural damage detection systems as a better data analysis scheme [9]. To this end, data mining has been employed as an extraction approach to acquire information on the structural health condition by means of classification-, prediction- and optimization-based data mining techniques [10].

Over the last decades, various optimization-based evolutionary algorithms have also been developed to solve a variety of engineering optimization problems in SHM, i.e. genetic algorithm [11], particle swarm optimization [12], ant colony optimization [13], artificial immune algorithm [14], firefly algorithm [15], artificial bee colony algorithm [16], grey wolf optimization [17] and imperialist competitive algorithm [18].

As mentioned earlier, a number of researchers have reported the applicability of data mining to solve SHM problems. It is also concluded that metaheuristic optimization-based techniques have so attracted a lot of attention from civil engineers. However, there is a lack of studies in the literature concerning specifically about optimization-based evolutionary techniques along with their applications in structural damage identification. Therefore, this paper provides a review of the development and implementation of metaheuristic techniques in SHM. 


\section{Metaheuristic techniques}

In real-life applications, there are lots of challenges and solution space is infinite or excessively huge in order to calculate all of the solutions. Consequently, with evaluating solutions, a reliable solution is required to be obtained in acceptable time. In fact, the assessment of solutions in acceptable time declares the similar importance with assessing "several solutions" in the whole solution space. Selection of several solutions is dependent on how and what they are picked changes based on metaheuristic techniques. Therefore, metaheuristic algorithms play a very important role in finding the optimized solutions. This is due to the fact that finding the exact solution is almost impossible for an optimization problem. Besides, in terms of comprehensibility, metaheuristic techniques are extensively straightforward in compare to decision makers. Another advantage of using these techniques is that they can be employed as a part of process of finding the exact solution, which means that they can also be combined by other algorithms.

Metaheuristics techniques can be classified in nine categories, i.e. biology based, social based, music based, physics based, chemistry based, swarm based, sport based, math based and hybrid based, as shown in Figure 1. Biology based techniques include Genetic Algorithm (GA) [11], Biogeography-Based Optimization (BBO) [19], Differential Evolution Algorithm (DEA) [20], Bacteria Foraging Optimization (BFO) [21], Artificial Immune Algorithm (AIA) [22] and Memetic Algorithm (MA) [23]. Imperialist Competitive Algorithm (ICA) [18], Teaching Learning Algorithm (TLA) [24], Parliamentary optimization algorithm [25] and Tabu Search (TS) [26] are Social based algorithms. Harmony Search (HS) [27] is a music based technique. Physics based methods consist of Intelligent Water Drops Algorithm (IWDA) [28], Gravitational Search Algorithm (GSA) [29], Charged System Search (CSS) [30], Big Bang-Big Crunch algorithm (BB-BC) [31] and Simulated Annealing Algorithm (SAA) [32]. Chemical Reaction Optimization (CRO) [33] represents the chemistry based category. There are various types of swarm based algorithms, i.e. Particle Swarm Optimization (PSO) [34], Monarch butterfly optimization [35], Cat Swarm Optimization (CSO) [36], Ant Colony Algorithm (ACO) [37], Group search optimizer [38], Bat Algorithm (BA) [39], Artificial Bee Colony Algorithm (ABCA) [16], Shuffled Frog Leaping Algorithm (SFLA) [40], Firefly Algorithm (FFA) [41], Grey wolf optimization (GWO) [42], Krill Herd (KH) [43], Monkey Algorithm [44], Intelligent Water Drops, Chaos Theory (CT) [45] and Mosquitoes Mating Strategy [46]. League championship algorithm [47] is considered a sport based metaheuristic technique. Base optimization algorithm (BOA) [48] is a math based approach. Last, but not least, hybrid based evolutionary algorithms are Cultural algorithm [49] and Colonial competitive differential evolution [50].

\section{Recent development of applicable optimization-based techniques in SHM}

\subsection{Particle Swarm Optimization (PSO)}

Particle Swarm Optimization (PSO), which is a population based optimization method, was first introduced by Kennedy and Eberhart [51]. This algorithm was inspired by the social behavior of organisms. For example, bird flocking can be employed as a suitable approach for global optimization. Particles in PSO stand for possible solutions. Each particle contains two updatable features; position, i.e. 'local' or 'global' best and velocity of each particle (see Figure 2). In PSO, the procedure starts with initializing the particles by means of their position as well as velocity. Then, the objective function is evaluated in order to update the velocity and position of particles with respect to the best fitness function. In the next step, it is required to determine the global minimum fitness value. After adjusting the velocity, the particle moves to its new position [52,53].

Structural damage detection based on improved PSO [54], damage identification of tensile test samples using hybrid PSO [55], optimization of support vector machine-based damage detection using PSO [56], damage identification of beams using fuzzy adaptive PSO [57], comparison of PSO and Big Bang-Big Crunch optimization damage detection method [31], and maximization of the probability density function utilizing hybrid PSO [58] are some of the most recent implementation and development of this technique.

As mentioned before, PSO has been used in SHM problems. For instance, a two-stage damage identification method based on evidence fusion and improved particle swarm optimization was proposed by [54]. In this direction, PSO was used to identify the extent of structural damage. The results showed that the proposed approach could successfully estimate the damage locations and extent with high accuracy.

PSO has been also developed for damage detection of beam-like structures by [57]. Herein, a hybrid approach comprising an improved PSO using nonlinearly decreasing inertia weight called NDW-PSO was proposed in this study. Moreover, a fuzzy adaptive PSO (APSO) were developed in a crack identification problem. Numerical simulation and experimental works were implemented on the damaged structure in order to verify the validity of the proposed methods. Figure 3 indicates the convergence trend of fitness values versus number of generations for different damage locations and corresponding damage severities. As it can be seen from this figure, the proposed APSO converged with less number of generations. NDW-PSO presented a better performance in compare to PSO. 
However, the results showed that the proposed APSO achieved the best performance among all algorithms in terms of percentage error. It was also concluded that APSO was successfully able to detect as well as locate the damage.

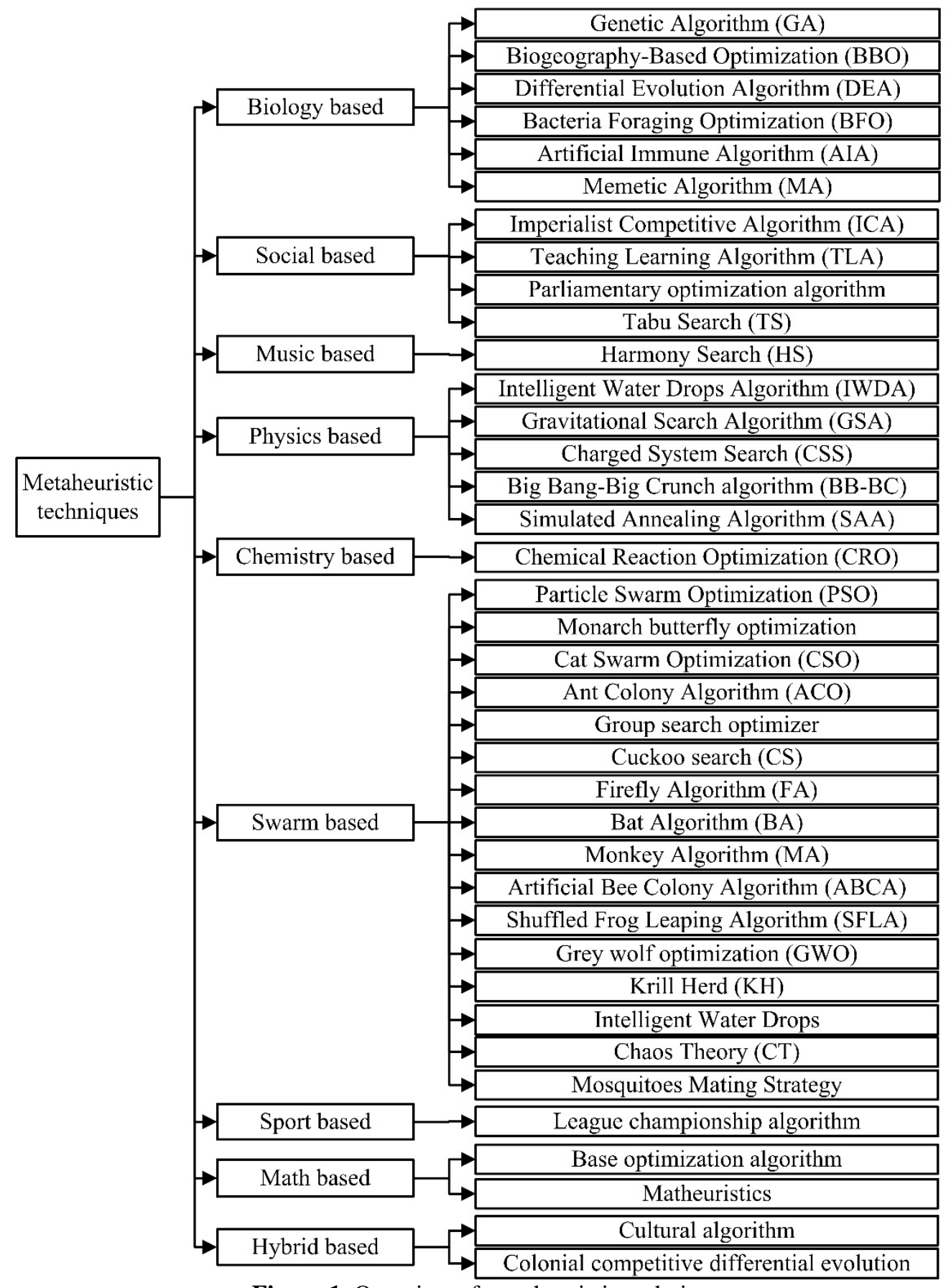

Figure 1. Overview of metaheuristic techniques

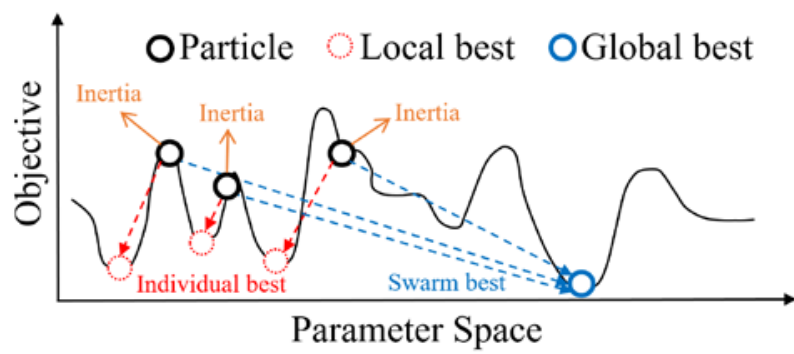

Figure 2. Overview of Particle Swarm Optimization 


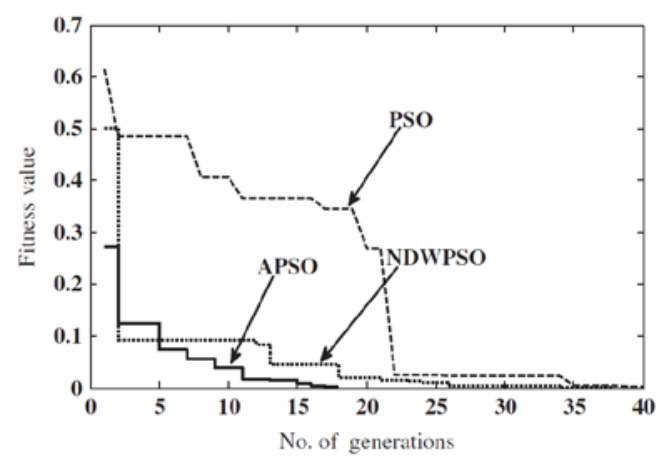

Figure 3. The convergence trend of fitness values against number of generations [57]

\subsection{Genetic Algorithm (GA)}

GA which was introduced by John Holland in 1970's, is one of the most common metaheuristic techniques. Genetic algorithm employs a set of genes, so-called chromosome in order to find the best solution by means of parameter optimization. In SHM, the parameters of any structural damage can be defined in a number of genes. Then, each individual solution is considered by creating its chromosome. Subsequently, the group of chromosomes form a population by analyzing the fitness of each chromosome. As shown in Figure 4, after selecting random solutions (parents) from population, two important operators (i.e. crossover and mutation) create the next generation [59,60]. Afterwards, it is required to calculate the fitness of new chromosomes. Last but not least, the same process which is selecting the parents' chromosomes and finding their fitness in the next generation will repeate, respectively until achieving a best solution [61].

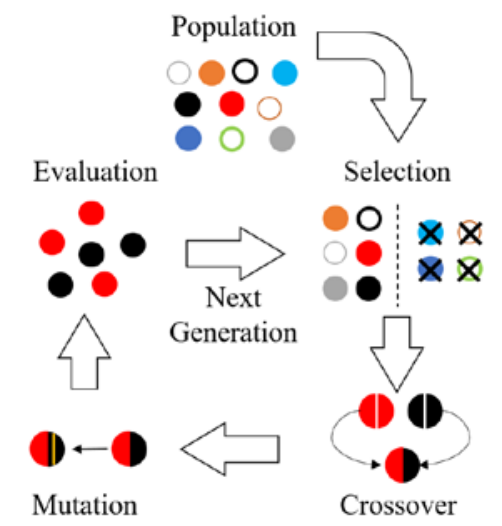

Figure 4. Schematic view of Genetic Algorithm

There are a variety of researchers focusing on the application of GA in SHM, i.e. damage detection of steel plates, beam-like structures, multistory structures and bridges [62-68]. It also applied to identify damage on dead load redistribution [69], Optimal sensor placement [70,71], search space reduction [72], fault detection filter [73], and vibration-based damage assessment of structures [74]

A hybrid approach based on Artificial Neuural Network (ANN) and GA for structural damage assessment of a three storey steel frame was presented by [63]. The results confirmed that the combined algorithm was an effective tool for damage identification and GA could improve the robustness of the pre-developed ANN. GA has been also used to improve the damage detection strategy of bridges by [65] using real-world data sets from the Z-24 Bridge, in Switzerland. In this work, the authors reported that the Expectation-Maximization (EM) algorithm could be applied in the parameter estimation of the Gaussian Mixture Models (GMMs) which corresponded to the normal and stable state conditions of the bridge. However, the performance of the EM algorithm was strongly dependent on the choice of the initial parameters. Therefore, a hybrid approach, called GA-EM-GMM based on a standard GA was proposed to improve the stability of the EM algorithm. The classification performance of EM-GMM and GA-EM-GMM were evaluated on the basis of type I/II error trade-offs, where a type I error was a false-positive indication of damage and a type II error was a false-negative one. Figure 5 is the performance of the EM-GMM approach over the executions. It was concluded that there were several extra repetitions in each execution in EMGMM. Hence, as shown in Figure 5, it was found that EM-GMM was quite unstable in compare to GA-EM-GMM in terms of performance. Besides, it was concluded that the GA-EM-GMM could achieve a better reliability and reproducibility due to its low standard deviation values for types I and II errors. 


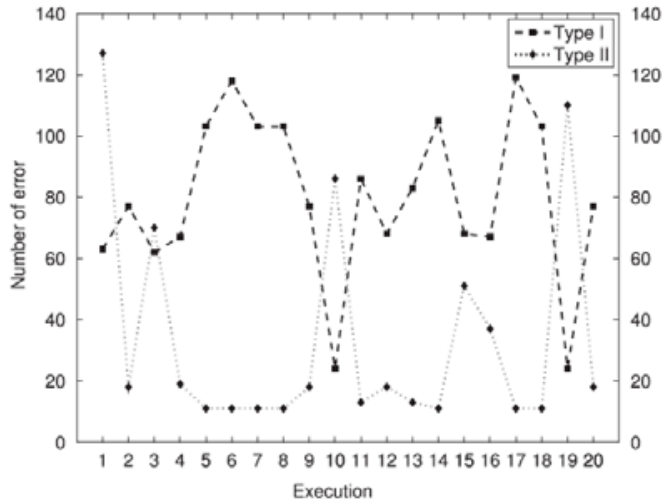

EM-GMM

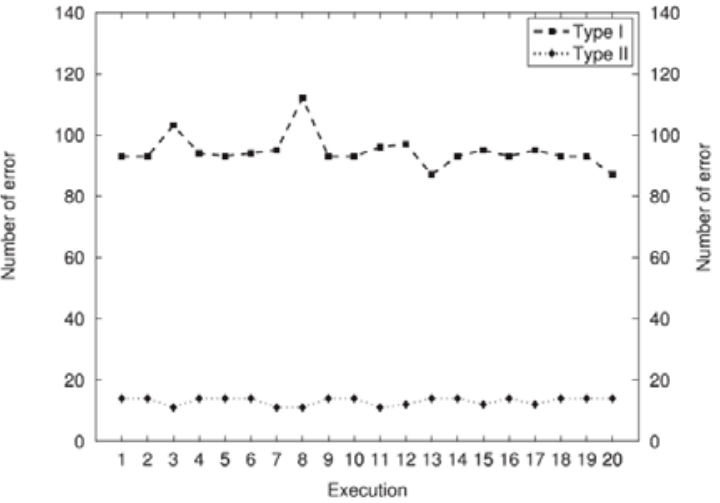

GA-EM-GMM

Figure 5. Classification performance of the EM-GMM and GA-EM-GMM

\subsection{Imperialist Competitive Algorithm (ICA)}

Imperialist competitive algorithm (ICA) was first proposed by Atashpaz-Gargari and Lucas in 2007 [75]. ICA is one of the recent metaheuristic optimization-based techniques that focused on human being's sociopolitical evolution. Finding a best solution which can represent a least possible value of fitness function or its global extreme value is the main key in this evolutionary computation algorithm [76]. ICA is based on the imperialistic competition. Therefore, it can start with initial populations called countries. Countries in ICA are corresponding to chromosomes in GA. In ICA, countries classify in two categorizes, i.e. imperialist and colony, which form an empire. According to the fundamental principles of optimization-based data mining techniques, the imperialists comprise countries with the least cost. Hence, the imperialist with maximum cost can be considered as the weakest imperialist. Figure 6 represents the overview of Imperialist Competitive Algorithm. As can be seen in this figure, the colony moves towards its proper imperialist.

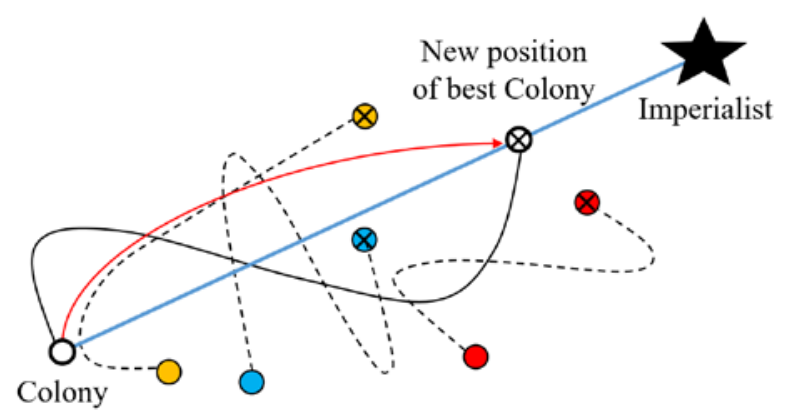

Figure 6. Overview of Imperialsit Competitive Algorithm

ICA has been used by [18] in order to improve the initial weights of ANN in the learning process. In this direction, the authors proposed a new data mining-based damage detection strategy along with a hybrid algorithm linking ICA and ANN, called ICA-ANN technique. Figure 7 demonstrates the network outputs versus the actual measured data for training and validation parts of the model. As it can be observed from the figure, red circles indicated the actual experimental results and blue squares denoted the ICA-ANN findings fitted to the real measured data, which evidenced the fitness of the proposed model. A comparison between the suggested model with a pre-developed neural network was conducted to validate the effectiveness of the developed approach. According to the obtained results, it was concluded that the ICA-ANN could indicate a better performance in detection of damage severity over the ANN method used only.

\subsection{Ant Colony Optimization (ACO)}

ACO was proposed by Marco Dorigo in 1992 for combinatorial optimization problems [37,77,78]. ACO is a probabilistic population-based algorithm inspired by the ants' foraging activities. The structure of ants communication is the main key in finding the best direction which can be achieved by pheromone trail [79-81]. ACO has been solved optimization problems through iterating two phases, i.e. constructing possible solutions in a probabilistic way by means of a probability distribution over the search space, and modification of the probability distribution by possible solutions [37]. ACO is organized in three configurations, i.e. ant-density, ant-quantity, and 
ant-cycle. Figure 8 shows the theory of ACO which is based on optimizing the best way to find the shortest path from nest to food source.
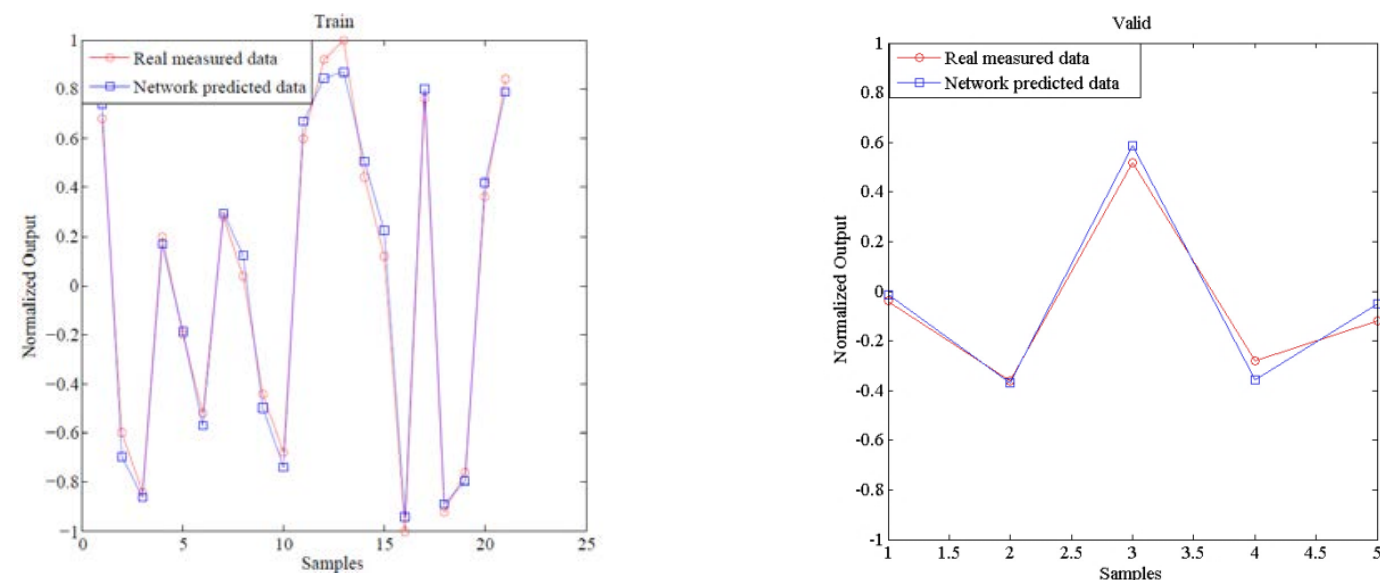

Figure 7. Reported results of ICA-ANN network by [18]
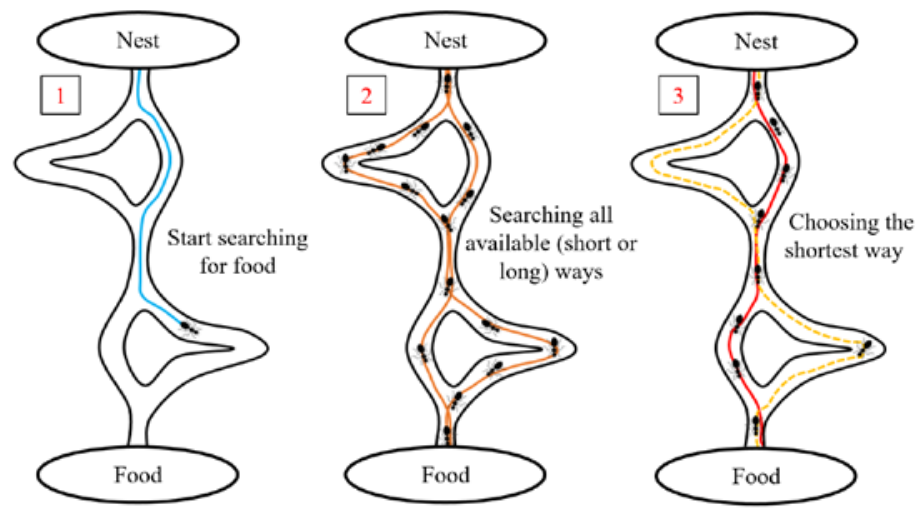

Figure 8. Theory of ACO

ACO has been also used in SHM, for example, the ACO based algorithm for optimization problems on structural damage detection [37], damage estimation of truss structures [81], sensor placement [82] and damage detection of a damped spring-mass system [83].

An optimized sensor placement strategy of Dalian North Bridge called the full-range pheromone ant colony system (FRPACS) was proposed by [82] based on the ACO to solve the placement of triaxle sensors. As can be seen from Figure 9, it was assumed that the trail of an ant was 1-31-12-14. The blue lines represent the trail of the ant. The pheromone laying method of the ACO and FRPACS are shown in the figure as well, where the green lines represent the pheromone laid by ant. The pheromone laying method of the ACO is revealed that the pheromone was laid on the path of 1-31, 31-12, 12-14. The authors believed that, if another ant is located in path No.1, it will be attracted by the pheromone to the trail of 1-31-12-14, and the optimal problem of the searching path could be solved by ACO. However, it was concluded that ACO was not effective enough in the optimal problem of selecting points. Therefore, the full-range pheromone ant colony system (FRPACS) was proposed to improve the iteration efficiency of the method. Based on the obtained results, it was reported that the FRPACS indicated a better performance in optimal sensor placement over the ACO method used only.

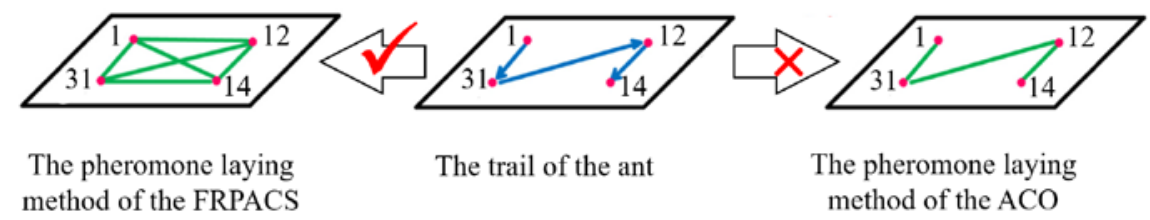

Figure 9. The comparison of the pheromone laying method between ACO and FRPACS 


\section{Conclusive remarks}

In general, the most challenging features of real world problems have been neglected in the definitions provided by numerical methods. Therefore, the lack of accuracy and efficiency of existing mathematical formulas have motivated researchers to work on evolutionary techniques established by nature inspired methods and their simulations to answer optimization problems in SHM. In addition, among the optimization-based algorithms, the metaheuristic techniques which were mostly identified as widespread principle optimization systems were known to be efficient in achieving the optimal solution to the real-valued problems. Therefore, these methods were effectively employed in optimization problems through linking facts and assumptions to simulate natural phenomena. The phenomena comprise the biological evolutionary procedure, e.g. GA, animal behavior e.g. PSO, the physical annealing, e.g. Simulated Annealing (SA) and etc.

This paper has presented the recent development of the most applicable optimization-based metaheuristic techniques used in structural damage identification. To aid the aim, Particle Swarm Optimization (PSO), Imperialist Competitive Algorithm (ICA), Genetic Algorithm (GA) and Ant Colony Optimization (ACO) along with their applications in SHM were reviewed. The process of implementing the PSO was quite easy due to its beneficial computational proficiency. The PSO also showed that it required a small volume of function evaluations to provide more accurate or the similar outcomes in compare to other optimization-based techniques. However, the PSO results revealed a few drawbacks due to its limitation in local optimization. In other words, PSO can be easily trapped in local optimum especially in multifaceted search spaces. Therefore, it is not advisable to employ this algorithm for a local optimum, since it cannot provide accurate local search near a local peak. In SHM, the key feature of PSO (optimization function) was helpful to evaluate the structural damage. Nevertheless, the implementation of PSO in damage identification of civil structures was less than GA applications. This is due to the fact that the search efficiency of PSO was not as attractive as GA. Hence, some improved strategies were presented, for instance, two convergence conditions, formula, micro-search of an elitist, mutation position iteration, etc.

GA was an ideal method in optimizing complicated difficulties due to its advantages, e.g. parallelism, adaptation and convergence to global optima. GA also proved that it was not required to involve the gradient of the objective function during the process. Accordingly, this evolutionary algorithm was effectively operated in SHM problems.

As mentioned before, various optimization-based biological evolution algorithms exist, including but not limited to artificial immune algorithm, GA, firefly algorithm, PSO, artificial bee colony algorithm, ACO, and grey wolf optimization. However, biological evolution was not the only metaheuristic strategy that has been used in SHM. In fact, evolutionary techniques comprise another approach inspired by humans' social political behavior besides biological systems which has been used as a solution for SHM problems. To this end, ICA has been proposed recently and presented its great achievements in terms of performance and accuracy. This is due to its abilities, i.e. quick convergence rate and high global optima in comparison to other metaheuristic techniques.

\section{Acknowledgement}

The authors would like to express their sincere thanks to University of Malaya (UM) and the Ministry of Higher Education (MOHE), Malaysia for the support given through research grant IIRG007A.

\section{References}

[1] Tan ZX, Thambiratnam DP, Chan TH, Razak HA. Detecting damage in steel beams using modal strain energy based damage index and Artificial Neural Network. Engineering Failure Analysis. 2017;79:253-262.

[2] Ghaedi K, Khanzaei P, Vaghei R, Fateh A, Javanmardi A, Gordan M, Hanif U. Reservoir hydrostatic pressure effect on roller compacted concrete (RCC) dams. Malaysian Construction Research Journal (MCRJ). 2016;19(2):1.

[3] Gordan M, Haddadiasl A, Marsono AK, Md Tap M. Investigation the behavior of a four-storey steel frame using viscous damper. Applied Mechanics and Materials. 2015;735:149-153.

[4] Ghayeb HH, Razak HA, Sulong NR. Development and testing of hybrid precast concrete beam-to-column connections under cyclic loading. Construction and Building Materials. 2017;151:258-278.

[5] Hou Z, Hera A, Noori M. Wavelet-based techniques for structural health monitoring. In: Heal. Assess. Eng. Struct. Bridg. Build. Other Infrastructures. World Scientific; 2013.p. 179-202.

[6] Gordan M, Ismail Z, Razak HA, Ibrahim Z. Vibration - based structural damage identification using data mining. In: 24th Int. Congr. Sound Vib. London; 2017.

[7] Adeli H. Sustainable infrastructure systems and environmentally-conscious design-A view for the next decade. Journal of Computing in Civil Engineering. 2002;16(4):231-233.

[8] Edeki CA. A comparative study of data mining and statistical learning techniques for prediction of canser 
survovability. Capella University; 2012.

[9] Duan Z, Zhang K. Data mining technology for structural health monitoring. Pacific Science Review. 2006;8(1):27-36.

[10] Gordan M, Razak HA, Ismail Z, Ghaedi K. Recent developments in damage identification of structures using data mining. Latin American Journal of Solids and Structures. 2017;14(13):2373-2401.

[11] Mitchell M. An introduction to genetic algorithms. MIT press; 1998.

[12] Xue B, Zhang M, Browne WN. Particle swarm optimization for feature selection in classification: A multiobjective approach. IEEE Transactions on Cybernetics. 2013;43(6):1656-1671.

[13] Zhou Y, Lai X, Li Y, Dong W. Ant colony optimization with combining Gaussian eliminations for matrix multiplication. IEEE Transactions on Cybernetics. 2013;43(1):347-357.

[14] Poteralski A, Szczepanik M, Dziatkiewicz G, Kuś W, Burczyński T. Comparison between PSO and AIS on the basis of identification of material constants in piezoelectrics. In:International Conference on Artificial Intelligence and Soft Computing. Springer, Berlin, Heidelberg. 2013. p. 569-581.

[15] Zhou GD, Yi TH, Li HN. Sensor placement optimization in structural health monitoring using cluster-incluster firefly algorithm. Advances in Structural Engineering. 2014;17(8):1103-1115.

[16] Civicioglu P, Besdok E. A conceptual comparison of the Cuckoo-search, particle swarm optimization, differential evolution and artificial bee colony algorithms. Artificial Intelligence Review. 2013;39(4):315346.

[17] Noshadi A, Shi J, Lee WS, Shi P, Kalam A. Optimal PID-type fuzzy logic controller for a multi-input multioutput active magnetic bearing system. Neural Computing and Applications. 2016;27(7):2031-2046.

[18] Gordan M, Razak HA, Ismail Z, Ghaedi K. Data mining based damage identification using imperialist competitive algorithm and artificial neural network. Latin American Journal of Solids and Structures. 2018;15(8): 1-14.

[19] Yang G, Zhang Y, Yang J, Ji G, Dong Z, Wang S, Feng C, Wang Q. Automated classification of brain images using wavelet-energy and biogeography-based optimization. Multimedia Tools and Applications. 2016;75(23):15601-15617.

[20] Lampinen J, Zelinka I. On stagnation of the differential evolution algorithm. In: Proc. MENDEL 2000, 6th Int. Mendel Conf. Soft Comput;2000. p.76-83.

[21] Abd-Elazim SM, Ali ES. Coordinated design of PSSs and SVC via bacteria foraging optimization algorithm in a multimachine power system. International Journal of Electrical Power \& Energy Systems. 2012;41(1):4453.

[22] Kalinli A, Karaboga N. Artificial immune algorithm for IIR filter design. Engineering Applications of Artificial Intelligence. 2005;18(8):919-929.

[23] Moscato P, Cotta C. A gentle introduction to memetic algorithms. In:Handbook of Metaheuristics. Springer, Boston, MA;2003. p. 105-144.

[24] Ghasemi M, Ghavidel S, Rahmani S, Roosta A, Falah H. A novel hybrid algorithm of imperialist competitive algorithm and teaching learning algorithm for optimal power flow problem with non-smooth cost functions. Engineering Applications of Artificial Intelligence. 2014;29:54-69.

[25] Borji A. A new global optimization algorithm inspired by parliamentary political competitions. In:Mexican International Conference on Artificial Intelligence 2007. Springer, Berlin, Heidelberg; 2007. p. 61-71.

[26] Abido MA. Optimal power flow using tabu search algorithm. Electric Power Components and Systems. 2002;30(5):469-483.

[27] Geem ZW, Kim JH, Loganathan GV. A new heuristic optimization algorithm: harmony search. Simulation. 2001;76(2):60-68.

[28] Shah-Hosseini H. The intelligent water drops algorithm: a nature-inspired swarm-based optimization algorithm. International Journal of Bio-inspired Computation. 2009;1(1-2):71-79.

[29] Sombra A, Valdez F, Melin P, Castillo O. A new gravitational search algorithm using fuzzy logic to parameter adaptation. In:2013 IEEE Congress on Evolutionary Computation; 2013.p. 1068-1074.

[30] Kaveh A. Applications of metaheuristic optimization algorithms in civil engineering. Switzerland: Springer; 2017.

[31] Tabrizian Z, Afshari E, Amiri GG, Beigy A, Hossein M, Nejad SM. A new damage detection method: Big Bang-Big Crunch (BB-BC) algorithm. Shock and Vibration. 2013;20(4):633-648.

[32] Heidari AA, Abbaspour RA, Jordehi AR. Gaussian bare-bones water cycle algorithm for optimal reactive power dispatch in electrical power systems. Applied Soft Computing. 2017;57:657-671.

[33] Alatas B. ACROA: artificial chemical reaction optimization algorithm for global optimization. Expert Systems with Applications. 2011;38(10):13170-1380.

[34] Peña E, Zhang S, Deyo S, Xiao Y, Johnson MD. Particle swarm optimization for programming deep brain stimulation arrays. Journal of Neural Engineering. 2017;14(1):016014.

[35] Wang GG, Deb S, Cui Z. Monarch butterfly optimization. Neural Computing and Applications. 
2019;31(7):1995-2014.

[36] Kumar GN, Kalavathi MS. Cat swarm optimization for optimal placement of multiple UPFC's in voltage stability enhancement under contingency. International Journal of Electrical Power \& Energy Systems. 2014;57:97-104.

[37] Yu L, Xu P. Structural health monitoring based on continuous ACO method. Microelectronics Reliability. 2011;51(2):270-278.

[38] He S, Wu QH, Saunders JR. Group search optimizer: an optimization algorithm inspired by animal searching behavior. IEEE Transactions on Evolutionary Computation. 2009;13(5):973-990.

[39] Gandomi AH, Yang XS, Alavi AH, Talatahari S. Bat algorithm for constrained optimization tasks. Neural Computing and Applications. 2013;22(6):1239-1255.

[40] Eghbal M, Saha TK, Hasan KN. Transmission expansion planning by meta-heuristic techniques: a comparison of shuffled frog leaping algorithm, PSO and GA. In: 2011 IEEE Power and Energy Society General Meeting; 2011.p. 1-8.

[41] Gandomi AH, Yang XS, Alavi AH. Mixed variable structural optimization using firefly algorithm. Computers \& Structures. 2011;89(23-24):2325-2336.

[42] Saremi S, Mirjalili SZ, Mirjalili SM. Evolutionary population dynamics and grey wolf optimizer. Neural Computing and Applications. 2015;26(5):1257-1263.

[43] Gandomi AH, Alavi AH. Krill herd: a new bio-inspired optimization algorithm. Communications in Nonlinear Science and Numerical Simulation. 2012;17(12):4831-4845.

[44] Yi TH, Li HN, Song G, Zhang XD. Optimal sensor placement for health monitoring of high-rise structure using adaptive monkey algorithm. Structural Control and Health Monitoring. 2015;22(4):667-681.

[45] Ghasemi M, Ghavidel S, Akbari E, Vahed AA. Solving non-linear, non-smooth and non-convex optimal power flow problems using chaotic invasive weed optimization algorithms based on chaos. Energy. 2014;73:340-353.

[46] Rathore C, Roy R, Raj S, Sinha AK. Mosquitoes-behaviour based (MOX) evolutionary algorithm in static transmission network expansion planning. In: 2013 International Conference on Energy Efficient Technologies for Sustainability; 2013.p. 1006-1011.

[47] Kashan AH. League championship algorithm: a new algorithm for numerical function optimization. In: 2009 International Conference of Soft Computing and Pattern Recognition; 2009. p. 43-48.

[48] Salem SA. BOA: A novel optimization algorithm. In: 2012 International Conference on Engineering and Technology (ICET); 2012. p. 1-5.

[49] Jin X, Reynolds RG. Using knowledge-based evolutionary computation to solve nonlinear constraint optimization problems: a cultural algorithm approach. In: Proceedings of the 1999 Congress on Evolutionary Computation-CEC99 (Cat. No. 99TH8406); 1999.Vol. 3, p. 1672-1678.

[50] Ghasemi M, Taghizadeh M, Ghavidel S, Abbasian A. Colonial competitive differential evolution: an experimental study for optimal economic load dispatch. Applied Soft Computing. 2016;40:342-363.

[51] Eberhart R, Kennedy J. Particle swarm optimization. In: Proceedings of the IEEE International Conference on Neural Networks; 1995. Vol. 4, p. 1942-1948.

[52] Talatahari S, Rahbari NM, Kaveh A. A new hybrid optimization algorithm for recognition of hysteretic nonlinear systems. KSCE Journal of Civil Engineering. 2013;17(5):1099-1108.

[53] Ghayeb HH, Razak HA, Sulong NH, Hanoon AN, Abutaha F, Ibrahim HA, Gordan M, Alnahhal MF. Predicting the mechanical properties of concrete using intelligent techniques to reduce $\mathrm{CO} 2$ emissions. Materiales de Construcción. 2019;69(334):e190.

[54] Guo HY, Li ZL. Structural damage identification based on evidence fusion and improved particle swarm optimization. Journal of Vibration and Control. 2014;20(9):1279-1292.

[55] Zhong J, Xu T, Guan K, Zou B. Determination of ductile damage parameters using hybrid particle swarm optimization. Experimental Mechanics. 2016;56(6):945-955.

[56] Gui G, Pan H, Lin Z, Li Y, Yuan Z. Data-driven support vector machine with optimization techniques for structural health monitoring and damage detection. KSCE Journal of Civil Engineering. 2017;21(2):523-534.

[57] Jena PK, Thatoi DN, Parhi DR. Dynamically self-adaptive fuzzy PSO technique for smart diagnosis of transverse crack. Applied Artificial Intelligence. 2015;29(3):211-232.

[58] Ng CT. Bayesian model updating approach for experimental identification of damage in beams using guided waves. Structural Health Monitoring. 2014;13(4):359-373.

[59] Kouchmeshky B, Aquino W, Billek AE. Structural damage identification using co-evolution and frequency response functions. Struct. Control Heal. Monit. 2008;15 (2): 162-182.

[60] Aghajanloo MB, Sabziparvar AA, Talaee PH. Artificial neural network-genetic algorithm for estimation of crop evapotranspiration in a semi-arid region of Iran. Neural Computing and Applications. 2013;23(5):13871393.

[61] Fu Z. Using genetic algorithms to develop inteligent decision trees. University of Maryland; 2000. 
[62] Rus G, Lee SY, Chang SY, Wooh SC. Optimized damage detection of steel plates from noisy impact test. International Journal for Numerical Methods in Engineering. 2006;68(7):707-727.

[63] Betti M, Facchini L, Biagini P. Damage detection on a three-storey steel frame using artificial neural networks and genetic algorithms. Meccanica. 2015;50(3):875-886.

[64] Mosquera V, Smyth AW, Betti R. Rapid evaluation and damage assessment of instrumented highway bridges. Earthquake Engineering \& Structural Dynamics. 2012;41(4):755-774.

[65] Santos A, Figueiredo E, Silva M, Santos R, Sales C, Costa JC. Genetic-based EM algorithm to improve the robustness of Gaussian mixture models for damage detection in bridges. Structural Control and Health Monitoring. 2017;24(3):e1886.

[66] Kuang KS, Maalej M, Quek ST. An application of a plastic optical fiber sensor and genetic algorithm for structural health monitoring. Journal of Intelligent Material Systems And Structures. 2006;17(5):361-379.

[67] Panigrahi SK, Chakraverty S, Mishra BK. Damage identification of multistory shear structure from sparse modal information. Journal of Computing in Civil Engineering. 2013;27(1):1-9.

[68] Liu H, Wang X, Gong Y, Jiao Y. Damage identification of urban overpass based on hybrid neurogenetic algorithm using static and dynamic properties. Mathematical Problems in Engineering. 2015;2015.

[69] Shenton III HW, Hu X. Damage identification based on dead load redistribution: methodology. Journal of Structural Engineering. 2006;132(8):1254-1264.

[70] Chen HG, Yan YJ, Chen WH, Jiang JS, Yu L, Wu ZY. Early damage detection in composite wingbox structures using Hilbert-Huang transform and genetic algorithm. Structural Health Monitoring. 2007;6(4):281-297.

[71] Cruz A, Vélez W, Thomson P. Optimal sensor placement for modal identification of structures using genetic algorithms - a case study: the olympic stadium in Cali, Colombia. Annals of Operations Research. 2010;181(1):769-781.

[72] Perry MJ, Koh CG. Output-only structural identification in time domain: numerical and experimental studies. Earthquake Engineering \& Structural Dynamics. 2008;37(4):517-533.

[73] Chen B, Nagarajaiah S. Observer-based structural damage detection using genetic algorithm. Structural Control and Health Monitoring. 2013;20(4):520-531.

[74] Srinivas V, Antony Jeyasehar C, Ramanjaneyulu K. Computational methodologies for vibration-based damage assessment of structures. International Journal of Structural Stability and Dynamics. 2013;13(08):1350043.

[75] Ebrahimi E, Mollazade K, Babaei S. Toward an automatic wheat purity measuring device: A machine visionbased neural networks-assisted imperialist competitive algorithm approach. Measurement. 2014;55:196-205.

[76] Geetha Devasena MS, Gopu G, Valarmathi ML. Automated and optimized software test suite generation technique for structural testing. International Journal of Software Engineering and Knowledge Engineering. 2016;26(01):1-3.

[77] Cottone G, Scimemi GF, Pirrotta A. $\alpha$-stable distributions for better performance of ACO in detecting damage on not well spaced frequency systems. Probabilistic Engineering Mechanics. 2014;35:29-36.

[78] Guerrero GD, Cecilia JM, Llanes A, García JM, Amos M, Ujaldón M. Comparative evaluation of platforms for parallel Ant Colony Optimization. The Journal of Supercomputing. 2014;69(1):318-329.

[79] Amini F, Ghaderi P. Optimal locations for MR dampers in civil structures using improved ant colony algorithm. Optimal Control Applications and Methods. 2012;33(2):232-248.

[80] Fidanova S, Marinov P, Alba E. Ant algorithm for optimal sensor deployment. In: Computational Intelligence 2012. Springer, Berlin, Heidelberg;2012. p. 21-29.

[81] Majumdar A, Maiti DK, Maity D. Damage assessment of truss structures from changes in natural frequencies using ant colony optimization. Applied Mathematics and Computation. 2012;218(19):9759-9772.

[82] Shuo F, Jingqing J. 3D sensor placement strategy using the full-range pheromone ant colony system. Smart Materials and Structures. 2016;25(7):075003.

[83] Braun CE, Chiwiacowsky LD, Gomez AT. Variations of Ant Colony Optimization for the solution of the structural damage identification problem. Procedia Computer Science. 2015;51:875-884.

(C) 2019 by the author(s). This work is licensed under a Creative Commons Attribution 4.0 International License (http://creativecommons.org/licenses/by/4.0/). Authors retain copyright of their work, with first publication rights granted to Tech Reviews Ltd. 